\title{
Sleep to be an All-Star!
}

\section{Kerstin Hödlmoser}

Nur ein ausgeruhter Athlet ist ein Sieger - Schlaf ist ein Grundbedürfnis und Lebenselixier. Ohne Frage ist erholsamer Schlaf die Voraussetzung für psychische und physische Leistungsfähigkeit. Doch im Leistungssport spielen die Erkenntnisse der Schlafforschung bislang nur eine untergeordnete Rolle. Dieser Artikel klärt über die Auswirkungen von Schlaf im Trainings- und Wettkampfprozess auf und liefert basale Kenntnisse über den normalen Schlaf und diverse im Leistungssport auftretende Schlafstörungen. Schließlich bietet er Tipps und Strategien, um das Schlafverhalten von Sportlern zu verbessern.

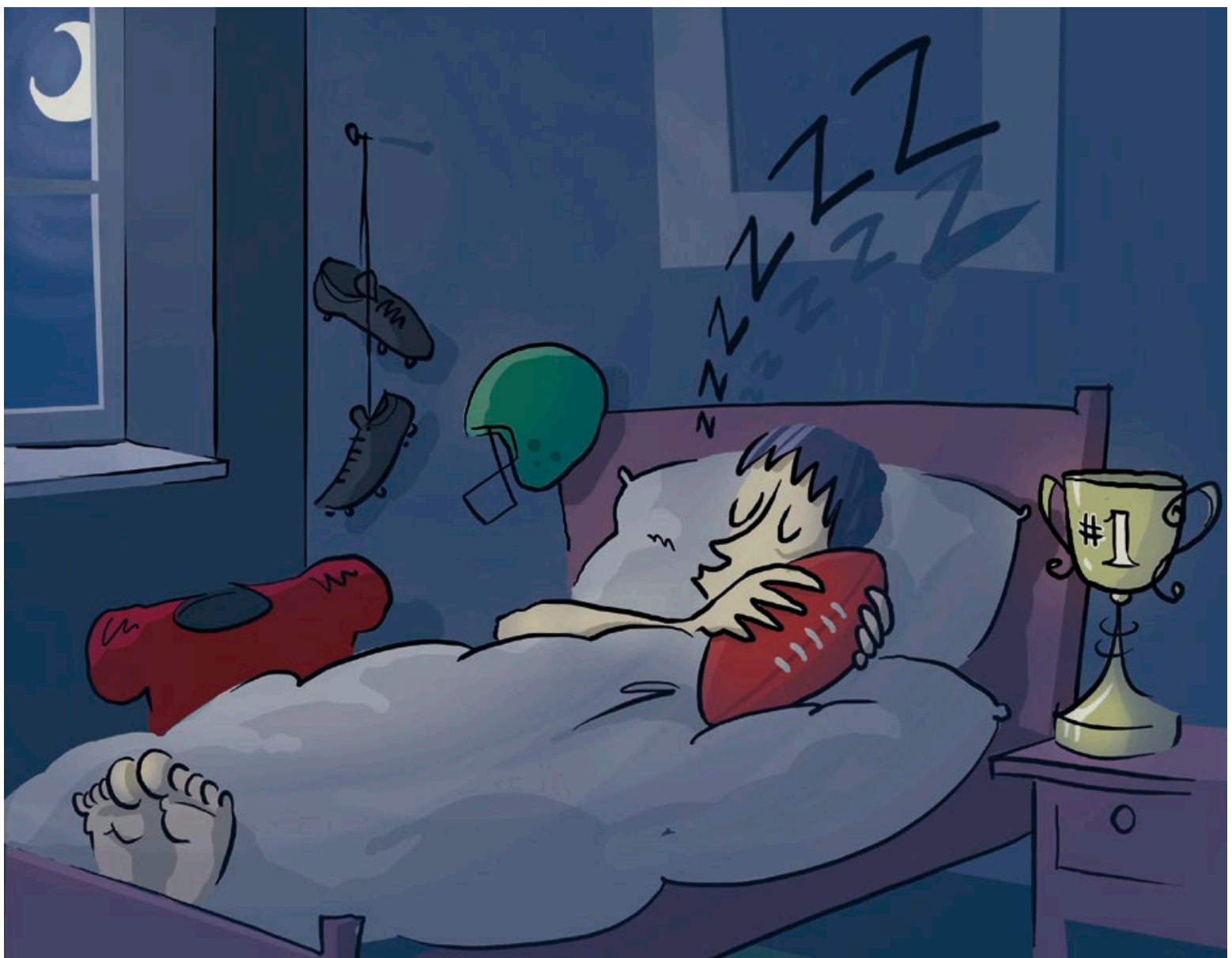

Abb. 1 Sleep to be an All-Star. (Quelle: Klaus Trifich, Salzburg)

Über den gesunden und gestörten Schlaf

\section{Wie viel Schlaf braucht der Mensch?}

Die durchschnittliche Schlafdauer eines gesunden Erwachsenen hat sich von ungefähr 8-9 Stunden pro Nacht im
Jahre 1959 auf 7-8 Stunden im Jahr 1980 reduziert [9]. Die „National Sleep Foundation for Healthy Sleep“ (2013) empfiehlt eine Schlafdauer von 7-9 Stunden. Zahlreiche Studien weisen darauf hin, dass die individuelle Schlafdauer sehr stark variiert [12], vor allem auch bei Athleten ( $\triangleright$ Abb. 2). Insbesondere Personen, die sich vor dem 


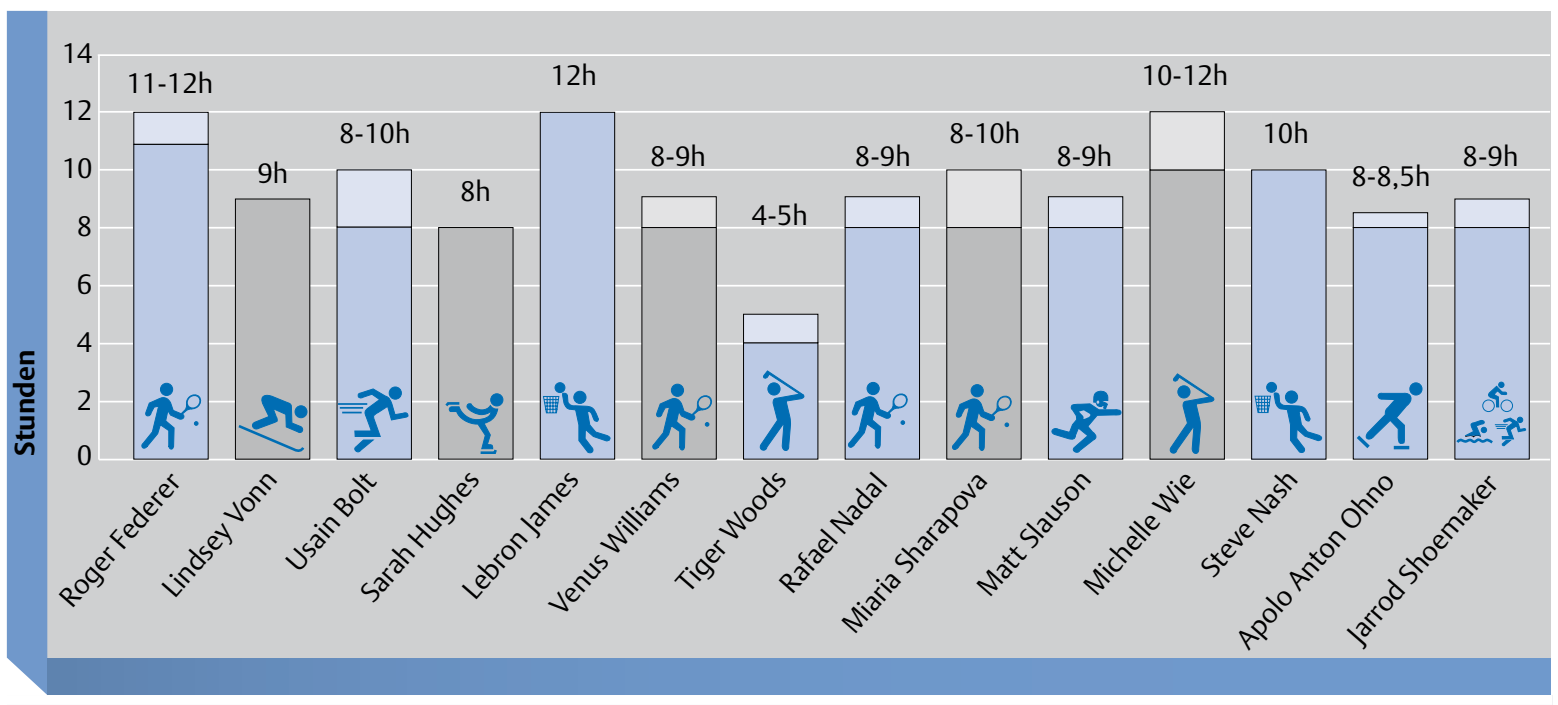

- Abb. 2 Wie viel Schlaf braucht ein Spitzensportler? (Quelle: K. Hödlmoser [modifiziert nach [25])

Zubettgehen mittels Smartphone, Laptops oder Tablets künstlichem Blaulicht aussetzen, zeigen eine stark reduzierte Schlafdauer. Unser Auge ist besonders empfindsam auf diese Lichtwellenlänge $(480 \mathrm{~nm})$ und leitet dadurch hormonelle Mechanismen (Unterdrückung des Hormons Melatonin) ein, die uns wach und aufmerksam machen, wodurch sich die Einschlafdauer verlängert. Es wird empfohlen, die eigene optimale Schlafdauer mittels Ausfüllen eines Schlaftagebuches (http://www.dgsm.de/schlaftagebuch) über den Zeitraum von mindestens zwei Wochen individuell zu ermitteln. Parallel zur Bestimmung des persönlichen Schlafverhaltens und der individuell optimalen Schlafdauer besteht des Weiteren die Möglichkeit, mittels Fragebogen („Morningness-Eveningness Questionnaire“ [14]) die individuelle zirkadiane Phasenlage (bin ich ein Morgen- oder Abendtyp?) zu identifizieren.

\section{LINKS}

Ermitteln der eigenen optimalen Schlafdauer das Schlaftagebuch:

http://bit.ly/2AIDHN4

\section{Wie kann man Schlaf messen?}

In der Schlafforschung wird der Schlaf anhand von drei physiologischen Parametern gemessen: den Gehirnströmen, den Augenbewegungen und dem Muskeltonus. Diese Parameter werden durch eine polysomnografische Aufzeichnung mittels Elektroenzephalografie (EEG), Elektrookulogramm (EOG) und Elektromyografie (EMG) erfasst. Über die Nacht hinweg zeigen sich zyklisch wiederholende Veränderungen in diesen physiologischen Parametern, wodurch man den Schlaf in verschiedene Stadien unterteilen kann [16]. Generell unterscheidet man zwischen NREM- (N1, N2, N3) und REM-Schlafstadien, die innerhalb von ca. 90-minütigen Phasen zyklisch aufeinan- der folgen. Der Nachtschlaf einer erwachsenen Person, die 8 Stunden schläft, setzt sich aus ungefähr 3-5 dieser Schlafzyklen zusammen. Das Schlafstadium N1 kennzeichnet den Übergang zwischen Wachheit und Schlaf, weshalb es auch als Einschlafstadium bezeichnet wird. Dieser Zustand wird häufig als „Dösen“ erlebt und umfasst in etwa $5 \%$ des Gesamtschlafes.

\section{"Sleep is extremely important to me - I need to rest and recover in order for the training I do to be absorbed by my body." \\ (Usain Bolt, Sprinter)}

Den prozentual größten Teil eines 8-stündigen Schlafes nimmt mit ca. $50 \%$ das Leichtschlafstadium N2 ein. Das Auftreten dieses Stadiums wird als eigentlicher Schlafbeginn angesehen, wobei der Schläfer aus diesem Stadium noch leicht erweckbar ist. Stadium N3 repräsentiert den Tiefschlaf und ist gekennzeichnet durch eine erschlaffte Muskulatur sowie langsame und regelmäßige Atem- und Herzfrequenz. Der Schläfer ist hier nur noch schwer erweckbar, und es bedarf bedeutender Signale von außen (beispielsweise das Hören des eigenen Namens), um ein Erwachen herbeizuführen. In diesem Schlafstadium verbringen wir ungefähr $20 \%$ unseres Schlafes. Der REMSchlaf (ca. $20 \%$ ) ist gekennzeichnet durch eine vollständige Muskelatonie, jedoch gleichzeitig eine wachähnliche kortikale Aktivierung und rasche, konjugierte Augenbewegungen (,rapid eye movements“, REM). Personen, die aus diesem Schlafstadium geweckt werden, erinnern sich häufig an lebhafte und unwirkliche Träume, weshalb der REM-Schlaf auch als Traumschlaf bezeichnet wird (wobei auch Träume aus anderen Schlafstadien in Erinnerung bleiben). Basierend auf der polysomnografischen Aufzeichnung kann der Verlauf dieser Schlafstadien als Schlafprofil 


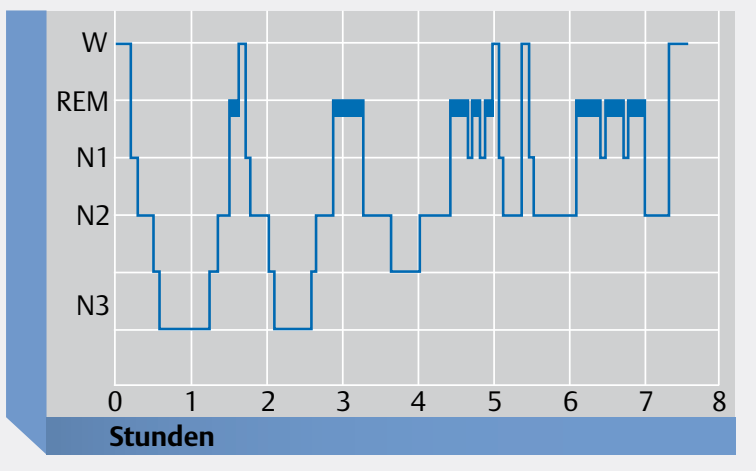

-Abb. 3 Schlafprofil eines gesunden Erwachsenen (Quelle: K. Hödlmoser)

\section{Insomnie}

A. Einschlaf-, Durchschlafschwierigkeiten, frühmorgendliches Erwachen und/ oder chronisch nicht erholsamer Schlaf

B. Die Schlafbeschwerde tritt auch trotz ausreichender Möglichkeiten zu schlafen und adäquaten

Rahmenbedingungen auf

C. Mindestens eine der folgenden Beschwerden wird berichtet:

Müdigkeit und allgemeines Unwohlsein

Aufmerksamkeits-, Konzentrations- und

Gedächtnisprobleme

Soziale, berufliche oder schulische Beeinträchtigung

Beeinträchtigung der Stimmung oder Irritierbarkeit

Tagesschläfrigkeit

Verringerung von Motivation, Antrieb oder Initiative

Erhöhte Anfälligkeit zu Fehlern und Unfällen im

Arbeitsumfeld oder Straßenverkehr

Angespanntheit, Kopfschmerzen oder gastrointestinale

Beschwerden als Folge des Schlafmangels

Sorgen bezüglich des Schlafverhaltens

\section{Anpassungsbedingte / akute Insomnie}

A. Die Beschwerden erfüllen die Kriterien der Insomnie

B. Die Schlafstörung steht im zeitlichen Zusammenhang mit einem identifizierbaren Stressor psychologischer,

psychosozialer, interpersoneller, umweltbedingter oder physischer Natur

C. Es kann erwartet werden, dass die Schlafstörung abklingt, wenn der Stressor wegfällt oder der Klient sich daran adaptiert

D. Die Schlafbeschwerden dauern weniger als 3 Monate

E. Die Schlafbeschwerden können nicht besser durch eine andere Schlafstörung, medizinische oder neurologische Erkrankungen, psychische Störungen,

Medikamenteneinnahme oder Substanzmissbrauch erklärt werden

- Abb. 4 Diagnosekriterien für Insomnie und „Anpassungsbedingte oder akute Insomnie" gemäß (International Classification of Sleep Disorders 2; American Academy of Sleep Medicine, 2005) (Quelle: K. Hödlmoser)

( $\triangleright$ Abb. 3) dargestellt werden. Betrachtet man dieses Profil, ist zu beobachten, dass der Tiefschlaf (N3) vor allem in der ersten und der REM-Schlaf eher in der zweiten Nachthälfte dominieren. Unser Schlaf-Wach-Rhythmus ist ein zirkadianer Rhythmus mit einer ungefähren Periodenlänge von 24 Stunden. Er wird zentralnervös gesteuert und unterliegt einer homöostatischen Regulation [3], dessen
Taktgeber (,innere Uhr“) im Nucleus suprachiasmaticus (eine Kernstruktur des Hypothalamus) sitzt. Neben dieser sogenannten „endogenen“ Steuerung des Schlafes spielen aber auch wesentliche „exogene“ Faktoren wie beispielsweise Licht, Umgebungstemperatur, Nahrung und körperliche Aktivität eine maßgebliche Rolle.

\section{„Exogene“ Faktoren wie beispiels- weise Licht, Umgebungstemperatur, Nahrung und körperliche Aktivität spielen bei der Steuerung des Schlafes eine maßgebliche Rolle.}

\section{Wozu schlafen wir?}

Dem Schlaf werden unterschiedliche Funktionen zugeordnet. Die zentralste Aufgabe ist mit Sicherheit die der Erholung und Regeneration. Eine Zunahme des Wachstumshormonspiegels während der ersten Nachthälfte sowie das Auffüllen der zerebralen Glykogenspeicher unterstützen diesen Ansatz [43]. Sowohl der Energieverbrauch als auch die Körpertemperatur sinken im Schlaf, was zusätzlich auf eine energiesparende Funktion schließen lässt. Insbesondere für den Athleten ist die Notwendigkeit des Schlafes für ein funktionstüchtiges Immunsystem hervorzuheben. Schlafdeprivationsstudien im Tier- und Humanbereich zeigen eine erhöhte Anfälligkeit für Infektionen bei reduzierter Schlafdauer [15]. Hinsichtlich eigener Forschungsbefunde gilt nicht zuletzt die Konsolidierung von neu erlernten Gedächtnisinhalten im Schlaf als wissenschaftlich gesichert [21]. Aufgaben, die vor einer Schlafperiode erlernt werden, können besser behalten werden als Aufgaben, die vor einer Wachperiode durchgeführt wurden. Diese Befunde stimmen auch mit den häufig beklagten Gedächtnis- und Konzentrationseinschränkungen von Sportlern mit beeinträchtigter Schlafqualität überein.

\section{Wann spricht man von einer Schlafstörung und welche Schlafstörungen gibt es?}

Nach der internationalen Klassifikation der Schlafstörungen [1] werden 81 Schlafstörungen unterschieden, welche folgenden 8 Gruppen zuordenbar sind:

1. Insomnien

2. Schlafbezogene Atmungsstörungen

3. Hypersomnien zentralnervösen Ursprungs

4. Zirkadiane Schlaf-Wach-Rhythmus-Störungen

5. Parasomnien

6. Schlafbezogene Bewegungsstörungen

7. Isolierte Symptome, Normvarianten und ungeklärte Beobachtungen

8. Andere Schlafstörungen

Dieser Vertiefungsbeitrag bezieht sich vorrangig auf die Klasse der Insomnien und hier insbesondere auf die „Anpassungsbedingte oder akute Insomnie“ ( Sportler während der Wettkampfphase, aber auch in der 
Vorbereitungsphase vor allem durch Nervosität, Leistungsdruck oder eine ungewohnte Schlafumgebung über Schlafstörungen klagen. Im Allgemeinen sind Insomnien durch eine erhöhte Einschlafzeit (> 30 min), vermehrte nächtliche Wachphasen und frühmorgendliches Erwachen bei ausreichender Bettzeit charakterisiert. Neben der gestörten Schlafqualität selbst wird des Weiteren eine subjektiv reduzierte Erholungsfunktion berichtet. Tageszeitliche Beeinträchtigungen wie Leistungseinschränkungen in Form von Aufmerksamkeits- und Gedächtnisstörungen sowie Müdigkeit, Erschöpfung, Mattigkeit, Gereiztheit, erhöhte Irritierbarkeit und sozialer Rückzug, bis hin zu somatischen Symptomen wie Muskelschmerzen, Kopfschmerzen und Magen-Darm-Beschwerden gelten als kennzeichnend. Eine Diagnose dieses Störungsbildes erfordert eine Mindestdauer von 4 Wochen [43]. Die „Anpassungsbedingte oder akute Insomnie “ ist durch einen spezifischen Stressor (z. B. ein bevorstehender wichtiger Wettkampf) ausgelöst und in der Regel von kurzer Dauer (Tage bis wenige Wochen; maximal drei Monate).

\section{„Sleep is half my training." \\ (Jarrod Shoemaker, Triathlet)}

Markant ist, dass diese Art von Insomnie remittiert, wenn die Belastung (im Falle eines Sportlers beispielsweise Leistungsdruck, Nervosität) wegfällt oder die Person sich an den Stressor angepasst hat. Treten die Beschwerden über mehr als 4 Wochen mindestens 3-mal pro Woche auf und führen sie zu Einschränkungen der Leistungsfähigkeit tagsüber, muss ein Arzt aufgesucht werden. Im diagnostischen Prozess ist zunächst der Ausschluss organischer oder psychischer Ursachen, die ebenfalls eine Insomnie hervorrufen können, unabdingbar. Die schlafmedizinische Diagnostik umfasst eine ausführliche Schlafanamnese inklusive Fremdanamnese in Bezug auf das nächtliche Schlafverhalten, ein Schlaftagebuch sowie gegebenenfalls verschiedene Schlaffragebögen ( $>$ Tab. 1). Eine stationäre Untersuchung mittels Polysomnografie im Schlaflabor zur Differentialdiagnose von beispielsweise periodischen Bewegungsstörungen im Schlaf oder schlafbezogenen Atmungsstörungen ist bei therapieresistenten Insomnien mit einer Dauer von mehr als 3-6 Monaten indiziert.

\section{Schlaf und Schlafstörungen im Spitzensport}

Aktuelle Studien zeigen, dass Athleten im Durchschnitt deutlich weniger schlafen (6-8 Stunden) als die für gesunde Erwachsene empfohlene Schlafdauer von 7-9 Stunden [23, 36]. Sowohl zu hoher Trainingsumfang [17, 31, 44] als auch starke Trainingsintensität unmittelbar vor dem Zubettgehen (40 Minuten Laufbandtraining bei $80 \%$ der HRmax um 21:30 Uhr [32]) wirkten sich auf die Schlafqualität negativ aus. Neben Belastungen zu später Stun-

\section{FAKTEN ÜBER SCHLAF UND KÖRPERLICHE}

\section{LEISTUNGSFÄHIGKEIT}

1. Eine Reduktion der Schlafzeit auf 4 Stunden über 6 Tage führte bei jungen Männern zu einer Verlangsamung des Glukosestoffwechsels um $40 \%$, einer Verringerung der Glukosewirksamkeit um $30 \%$ sowie einer reduzierten Insulinantwort, vergleichbar mit Werten bei Diabeteserkrankten oder in hohem Alter. Des Weiteren zeigten sich erhöhte Kortisolwerte am Abend aufgrund eines verlangsamten Kortisolabbaus und eine erhöhte sympathische Aktivität [41].

2. Tennisspielerinnen, die ihre Schlafdauer über 5-6 Wochen auf 10 Stunden erhöhten, zeigten neben einer positiveren Stimmung und schnelleren Sprintleistung eine um $42 \%$ erhöhte Treffgenauigkeit, insbesondere bei „depth drills“ [27].

3. 36 Stunden ohne Schlaf führten bei gesunden jungen Männern zu einer um $11 \%$ schnelleren subjektiven Erschöpfung während eines Laufbandtestes, wobei die objektiven Parameter (z. B. Herz- und metabolische Rate) unverändert blieben [29].

4. Schwimmer und Schwimmerinnen, die über 5-6 Wochen ihre Schlafzeit auf 10 Stunden erhöhten, zeigten neben einer schnelleren Sprintleistung und verbesserten Stimmung eine um $17 \%$ schnellere Abstoßreaktion vom Startblock sowie eine um $10 \%$ schnellere Wende [26].

5. Eine Reduktion der individuellen Schlafdauer um $50 \%$ über 7 Tage resultierte bei gesunden Männern und Frauen in einer Zunahme der subjektiven Schläfrigkeit sowie einer Abnahme des subjektiv eingeschätzten Elans. Darüber hinaus zeigte sich eine Zunahme der Reaktionszeit sowie der Anzahl an Fehlern während einer Daueraufmerksamkeitsaufgabe. Diese Defizite konnten erst nach 2 Tagen Erholungsschlaf ausgeglichen werden [6].

6. Eine Nacht an erholsamen Schlaf führte in einer Kategorisierungsaufgabe zu einer Erhöhung der Genauigkeit der Informationsintegrationsfähigkeit um 4,3\%. Hingegen resultierten 24 Stunden Schlaflosigkeit in einer Leistungsreduktion um 2,4\% [24].

7. Eine 2-tägige Reduktion der Schlafdauer auf 3 Stunden führte bei jungen Männern zu einer reduzierten Leistungsfähigkeit um 3lbs (1,36 kg) beim Gewichtheben [34].

8. American-Football-Spieler, die ihre Schlafzeit auf mindestens 10 Stunden über einen Zeitraum von 7-8 Wochen erhöhten, zeigten neben reduzierter Müdigkeit und gesteigertem Elan eine Verbesserung um 0,1 Sekunde im 20-Yard-Shuttle und im 40-Yard-Sprint [28].

9. 30 Stunden Schlaflosigkeit resultierten in einer Erhöhung der subjektiv wahrgenommenen Kraftanstrengung um 17-19\% in einem 8-minütigen Ergometertraining sowohl bei mittlerer als auch bei starker Beanspruchung, wobei die physiologischen Parameter sich nicht veränderten [30].

10. Ein 10-minütiges Nickerchen steigerte neben der subjektiv empfundenen Wachheit auch die objektive Wachheit [45]. 
de scheinen auch Trainingszeiten am frühen Morgen den Schlaf zu beeinträchtigen [36]. Der Kasten „10 Fakten über Schlaf und körperliche Leistungsfähigkeit“ bietet eine Faktenübersicht basierend auf Studien, welche sich speziell mit den Auswirkungen von Schlaf auf die sportliche Leistungsfähigkeit beschäftigen (modifiziert nach [25]). Je nach Ausprägungsgrad der gegebenenfalls entwickelten Schlafstörung berichten Athleten teilweise erhebliche Beeinträchtigungen und fühlen sich außerstande, ihre optimale Wettkampfleistung zu erbringen. Entsprechend einer Vielzahl an Untersuchungen $[2,10,11,19]$ berichten Athleten sehr häufig, dass sie vor einem Wettkampf mit Einschlafschwierigkeiten, frühmorgendlichem und nächtlichem Erwachen konfrontiert sind. Des Weiteren konnten Erlacher, Ehrlenspiel und Schredl [8] aufzeigen, dass Athleten vor einem Wettkampf vermehrt mit Angstträumen über den eigenen sportlichen Misserfolg konfrontiert sind. Derartige Angstträume können ebenfalls starke negative Effekte auf das emotionale Tagesbefinden am Wettkampftag (z. B. Selbstvertrauen, Siegeswillen) und somit auf die sportliche Leistungsfähigkeit haben.

\section{„Eat, sleep and swim, that's all I can do." \\ (Michael Phelps, Schwimmer)}

Als Ursachen für den schlechten Schlaf vor einem Wettkampf wurden in der Studie von Erlacher und Kollegen [8] von mehr als der Hälfte der befragten 632 deutschen Kaderathleten „Gedanken über den Wettkampfverlauf“ sowie „Nervosität vor dem Wettkampf“ berichtet. Neben dem direkten Einfluss der reduzierten Schlafqualität auf die sportliche Leistungsfähigkeit berichten Steininger und Wodick [42], dass Athleten bereits durch das alleinige Bewusstsein über ihr schlechtes Schlafverhalten (beispielsweise ausgelöst durch Übererregung, Nervosität oder Wettkampfangst) direkt vor dem Wettkampf, aber auch in der Wettkampfvorbereitungsphase selbst zu nervösen Überreaktionen, Fehlentscheidungen und zu früherer Resignation im Wettkampf tendieren $[35,44]$. Neben den bisher genannten psychischen Faktoren spielen im Spitzensport zusätzlich sehr häufig das Schlafen in ungewohnter und fremder Umgebung sowie die Anpassung an eine andere Zeitzone eine wesentliche Rolle für das beeinträchtigte Schlafverhalten der Athleten. In einer Untersuchung von Volleyballspielerinnen zu ihrem Schlafverhalten vor Wettkämpfen [7] wurden beispielsweise Verkehrslärm und unbequeme Betten als externe Störfaktoren genannt. Sowohl Wettkämpfe in anderen Zeitzonen oder zu ungewöhnlichen Tageszeiten, die Wettkampfanreise selbst als auch die Ernährung sowie ungewohnte geografische Gegebenheiten wie Höhe [37], Temperatur oder Luftfeuchtigkeit können zu einer anpassungsbedingten Insomnie führen [38]. Wissenschaftliche Befragungen deuten darauf hin, dass Athleten und ihr Betreuerstab nur über wenige bzw. unzureichende Strategien im Umgang mit Schlaf- problemen vor, während und auch nach einem Wettkampf wissen. Im Folgenden wird ein Überblick über mögliche Verhaltensmaßnahmen, insbesondere bei reduzierter Schlafqualität - mit speziellem Fokus auf Schlafstörungen im Zusammenhang mit einem Zeitzonenwechsel -, präsentiert.

$$
\begin{aligned}
& \text { "If I don't sleep 11-12 hours a day, } \\
& \text { it is not right." } \\
& \text { (Roger Federer, Tennisspieler) }
\end{aligned}
$$

\section{Jetlag}

Jetlag bezeichnet eine Schlafstörung, die bei einem Zeitzonenwechsel auftreten kann, und zählt zu den Störungen des zirkadianen Schlaf-Wach-Rhythmus, die sich auf die Verteilung des Schlafes innerhalb eines 24-Stunden-Tages beziehen [1]. Gekennzeichnet ist dieses Störungsbild durch unterschiedlich stark ausgeprägte Ein- oder Durchschlafschwierigkeiten, übermäßige Schläfrigkeit tagsüber, verminderte Wachheit und Leistungsfähigkeit am Tage sowie vegetative Symptome (z. B. veränderter Appetit, Veränderung gastrointestinaler Funktionen, Zunahme der Häufigkeit nächtlichen Wachwerdens, um Wasser zu lassen). Neben einer Störung des Schlaf-Wach-Rhythmus können auch andere Körperfunktionen (z. B. Körpertemperatur, Verdauung), die dem zirkadianen Rhythmus unterliegen, beeinträchtigt sein. Außerdem können sich Symptome wie Kopfschmerzen und Schwindel bemerkbar machen. Die Flugrichtung spielt für die Entstehung von Jetlag-Symptomen eine wichtige Rolle [22]. Generell sind Flüge nach Westen, bei denen sich der Tag verlängert, für den Körper besser verträglich als Flüge gen Osten, bei denen der Tag kürzer wird. Dies liegt daran, dass unsere innere Uhr nicht genau auf 24 Stunden, sondern in etwas längeren Taktphasen läuft. Flüge nach Westen kommen der inneren Uhr deshalb eher entgegen. Wenn man beispielsweise von Frankfurt am Main in die USA nach San Francisco fliegt und dort um 18 Uhr landet, ist es in Deutschland Mitternacht. Bleibt man nun noch ein paar Stunden wach, fällt man sehr erschöpft und müde ins Bett, kommt aber gut in den neuen Schlaf-Wach-Rhythmus hinein. Fliegt man hingegen von San Francisco nach Frankfurt am Main mit einer Ankunftszeit um 18 Uhr, ist der Körper noch an die Zeit in San Francisco (hier ist es erst 12 Uhr) gewöhnt. Deswegen fällt es einem hier in der Regel schwerer, in ein paar Stunden bereits schlafen gehen zu können. Je mehr Zeitzonen überflogen werden, desto intensiver ist die Symptomatik. Generell gilt, dass man pro Zeitzone mit einer Anpassungszeit von ungefähr einem Tag rechnen muss, wobei hier auch der Gesundheitszustand und das Alter des Reisenden eine Rolle spielen. Insbesondere für Athleten, die häufig Zeitzonen wechseln müssen, ist es empfehlenswert, sich frühzeitig an die Zeit im Zielland zu adaptieren. Bei einer Zeitverschiebung von 6 Stunden sollte man im Idealfall mindestens 6 Tage vor dem Wettkampf anreisen. Ist eine derartige Adaptionsphase nicht möglich, 
- Tab. 1 Subjektive Messinstrumente im Rahmen der Schlafanamnese

\begin{tabular}{|l|l|l|l|}
\hline Messverfahren & Einsatzbereich & Dauer & Autoren \\
\hline Pittsburgh Schlafqualitätsindex & Schlafqualität in den letzten 4 Wochen & $5-10$ Min & Buysse et al., 1991 \\
\hline Epworth Sleepiness Scale & Tagesmüdigkeit/-schläfrigkeit & 5 Min & Johns, 1991 \\
\hline Morningness-Eveningness-Fragebogen & Chronotyp & $5-10$ Min & Horne \& Östberg, 1976 \\
\hline Erholungs-Belastungs-Fragebogen & Aktuelle Beanspruchungs-Erholungs-Bilanz & $5-10$ Min & Kallus, 1995 \\
\hline Abend- \& Morgenprotokoll & Schlaftagebuch, Schlafverhalten & 5 Min & Hoffmann, Müller, Hajak, \& Cassel, 1997 \\
\hline
\end{tabular}

kann man bereits im Heimatland bestimmte Vorkehrungen treffen. Bei Flügen nach Osten sollte man jeweils eine Stunde früher schlafen gehen und eine Stunde früher aufstehen. Bei Flügen in den Westen genau umgekehrt. Eine vorzeitige Anpassung mittels zeitlich geplanter Lichteinwirkung (beispielsweise Lichtstimulation durch eine Tageslichtlampe) zur Unterdrückung der Melatonin-Ausschüttung und gleichzeitig zur Erleichterung der Wachheit kann unterstützend angewandt werden. In den frühen Abendstunden sollte man Licht aufsuchen, wohingegen man in der 2. Nachthälfte sowie am frühen Morgen Licht vermeiden sollte. Sonnenbrillen können genutzt werden, um die Lichteinwirkung zu reduzieren. Es wird empfohlen, den Flug so zu buchen, dass man am Nachmittag im Zielland ankommt, damit man in absehbarer Zeit schlafen gehen kann. Während des Fluges wird empfohlen, etwaige Uhren (Armband, Smartphone) auf die Zeit im Zielland umzustellen und sich bereits während dem Flug entsprechend der Zeit im Zielland zu verhalten. Beispielsweise servieren die Fluggesellschaften mittlerweile weitgehend die Mahlzeiten entsprechend dieser Empfehlungen. Während dem Flug sollte man auf ausreichend Hydration achten, jedoch Koffein und Alkohol vermeiden. Im Zielland angekommen, können kurze (maximal 20 Minuten) Nickerchen zur Erholung nach Schlafdeprivation beitragen. Natürliches Licht ist dem künstlichen Licht immer vorzuziehen und hilft ebenfalls bei der Umstellung des Schlaf-Wach-Rhythmus.

\section{Strategien und Tipps, um Schlafqualität bei Sportlern zu verbessern}

Klagt ein Sportler über vorübergehende (weniger als 4 Wochen) Schlafprobleme, sollte zunächst im ersten Schritt ein Schlafprotokoll eingesetzt werden (s. • Link-Kasten: Fragebogen zum Chronotyp (D-MEQ) DGSM-Schlafprotokoll). Ein Schlafprotokoll dient dazu, die individuellen (eventuell gestörten) Schlafgewohnheiten zu bestimmen. Zur Feststellung der eigenen individuellen Schlafdauer sollte man zumindest eine Woche jeden Tag zur glei-

\section{LINKS}

Fragebogen zum Chronotyp (D-MEQ):

http://bit.ly/2i4621Y chen Zeit ins Bett gehen und ohne Wecker ausschlafen. Bis zum 4. Tag sollte sich die persönliche Schlaflänge einpendeln. In Tabelle 1 ist eine Reihe von standardisierten Schlaffragebögen zur Selbstbeurteilung der Schlafqualität und -quantität (Pittsburgh Schlafqualitätsindex) sowie der Tagesmüdigkeit („Epworth Sleepiness Scale“ zur Erfassung der Tagesmüdigkeit/-schläfrigkeit) als auch der aktuellen Belastungssituation (Erholungs-Belastungs-Fragebogen) und Identifizierung des Chronotyps (Morgentyp-/Abendtyp-Fragebogen) aufgelistet. Diese Fragebögen ermöglichen eine Objektivierung der Schlafproblematik und können gegebenenfalls den diagnostischen Prozess bei einer Schlafstörung wesentlich vereinfachen und ökonomischer gestalten.

\section{TAKE HOME MESSAGE}

\section{Schlafhygiene-Regeln für Sportler:}

- Ermitteln Sie mithilfe eines Schlafprotokolls Ihre individuelle Schlafdauer.

- Halten Sie jeden Tag (auch am Wochenende) regelmäßige Aufsteh- und Zubettgehzeiten ein.

- Wenn Sie einen Mittagsschlaf halten, achten Sie darauf, dass dieser vor 15 Uhr liegt und maximal 20-30 Minuten dauert, um Tiefschlafphasen zu vermeiden.

- Schaffen Sie sich ein gemütliches und adäquates Schlafambiente: störende Licht- und Lärmquellen ausschalten sowie extreme Temperaturen vermeiden (eine Raumtemperatur von $18 \mathrm{Grad}$ ist ideal).

- keine schweren Mahlzeiten vor dem Zubettgehen

- keine koffeinhaltigen Getränke nach 18:00 Uhr

- 60 Minuten vor dem Zubettgehen keine körperlich oder geistig anstrengenden Aktivitäten

- Überlegen Sie sich Ihr persönliches 30-45-minütiges Einschlafritual (leichtes Stretching, Yoga, Lesen - Printlektüre, nicht am Tablet, um Lichteinflüsse zu vermeiden, Musikhören, warmes Duschen etc.).

- Das Bett ist nur zum Schlafen da (Ausnahme: sexuelle Aktivität) - der Körper soll lernen, dass er im Bett zur Ruhe findet.

- Helles Licht ist ein „Wachmacher“. Achten Sie darauf, dass Sie sich vor dem Einschlafen und nachts keinem hellen Licht aussetzen $\rightarrow$ nicht mit dem Smartphone, Tablet usw. ins Bett! Wenn, dann verwenden Sie den „Nachtmodus“ (Blaulichtfilter). 
- Wenn Sie sich in der Früh oder tagsüber erschöpft fühlen, gehen Sie ans Tageslicht oder verwenden Sie eine Tageslichtlampe.

- Planen Sie an stressigen, trainingsintensiven Tagen tagsüber Phasen der Regeneration (Entspannungsübungen, Nickerchen, Musikhören etc.) ein.

- Schreiben Sie vor dem Schlafen oder wenn Sie nachts aufwachen und nicht mehr einschlafen können, alles nieder, was Sie bewegt oder was Sie erledigen wollen. Das macht den Kopf frei!

- Wenn Sie nicht einschlafen können, vermeiden Sie negative Gedanken und machen Sie sich bewusst locker. Gehen Sie dafür gedanklich jeden Muskel durch und lockern Sie ihn. Vergessen Sie hier auch nicht die Gesichtsmuskulatur! Mit einem Stirnrunzeln schläft niemand gut ein!

- Wenn Sie nachts erwachen und nicht mehr einschlafen können, setzen Sie sich nicht unter Druck. Anstatt sich zu ärgern, dass Sie nicht schlafen können, denken Sie lieber: „Wie schön, dass ich noch nicht aufstehen muss." Genießen Sie die verbleibende Zeit - oft erleichtern positive Gedanken das Einschlafen.

\section{LINKS}

- Schlaflabor-Homepage der Salzburger Universität mit aktuellen Publikationen und Infos aus unserem Lab: www.sleepscience.at

- Projekthomepage von Kerstin Hödlmoser mit Infos zu Kinderschlaf: www.fit4schol.or.at

\section{Autorinnen/Autoren}

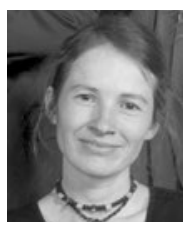

\section{Kerstin Hödlmoser}

ist Assistenzprofessorin an der Universität Salzburg und forscht seit 2002 zum Thema Schlaf und kognitive Leistung vom Kindes- bis ins Erwachsenenalter. Nebenbei ist sie Klinische und Gesundheitspsychologin sowie Sportpsychologin und Verhaltenspsychotherapeutin in Ausbildung unter Supervision.

\section{Korrespondenzadresse}

\section{Ass. Prof.-Dr. Kerstin Hödlmose}

Universität Salzburg, Fachbereich Psychologie, Centre for Cognitive Neuroscience

Labor für Schlaf, Kognition und Bewusstseinsforschung Hellbrunnerstr. 34, A-5020 Salzburg

E-Mail: kerstin.hoedlmoser@sbg.ac.at

https://ccns.sbg.ac.at/people/hoedlmoser/

\section{Literatur}

[1] American Academy of Sleep Medicine. International Classification of sleep disorders, $2^{\text {nd }}$ ed.: Diagnostic and coding manual. Westchester, Illinois: American Academy of Sleep Medicine, 2005
[2] Anglem N, Lucas SJ, Rose E A et al. (2008). Mood, illness and injury responses and recovery with adventure racing. Wilderness \& Environmental Medicine 2008; 19: 30-38

[3] Borbély A. Schlaf als regulierter Prozess. In H. Schulz, P. Geisler \& A. Rodenbeck (Eds.), Kompendium Schlafmedizin 7 (Kap. I-0), Landsberg: ecomed Medizin 2004

[4] Buysse DJ, Reynolds III CF, Monk TH et al. Quantification of subjective sleep quality in healthy elderly men and women using the Pittsburgh Sleep Quality Index (PSQI). Sleep 1991; 14: $331-338$

[5] Dement WC. Sleep extension: Getting as much extra sleep as possible. Clinics in Sports Medicine 2005; 24: 251-268

[6] Dinges DF, Pack F, Williams K et al. Cumulative sleepiness, mood disturbance, and psychomotor vigilance performance decrements during a week of sleep restricted to $4-5$ hours per night. Sleep 1997; 20: 267-277

[7] Erlacher D, Schredl M, Lakus G. Subjective sleep quality prior to home and away games for female volleyball players. Universitätsbibliothek der Universität Heidelberg 2009

[8] Erlacher D, Ehrlenspiel F, Schredl M. Frequency of nightmares and gender significantly predict distressing dreams of German athletes before competitions or games. The Journal of Psychology 2011; 145: 331-342

[9] Ferrara M, De Gennaro L. How much sleep do we need? Sleep Medicine Reviews 2001; 5: 155-179

[10] Fietze I, Strauch J, Holzhausen M et al. Sleep quality in professional ballet dancers. Chronobiology International 2009; 26: 1249-1262

[11] Forndran, A., Lastella M, Roach GD et al. Training schedules in elite swimmers: No time to rest. Sleep of different populations. Adelaide: Australasian Chronobiology Society 2012; 6-10

[12] Fullagar HH, Skorski S, Duffield R et al. Sleep and athletic performance: The effects of sleep loss on exercise performance, and physiological and cognitive responses to exercise. Sports Medicine 2015; 45: 161-186

[13] Hoffmann RM, Müller T, Hajak G et al. Abend-Morgenprotokolle in Schlafforschung und Schlafmedizin - Ein Standardinstrument für den deutschsprachigen Raum. Somnologie 1997; 1: 103-109 (http://www.dgsm.de/downloads/fachinformationen/frageboegen/2wochen.pdf)

[14] Horne JA, Östberg O. A self-assessment questionnaire to determine morningness-eveningness in human circadian rhythms. International Journal of Chronobiology 1976; 4 : 97-110

[15] Ibarra-Coronado EG, Pantaleón-Martínez AM, Velázquez-Moctezuma J et al. The bidirectional relationship between sleep and immunity against infections. Journal of Immunology Research 2015

[16] Iber C, Ancoli-Israel S, Chesson A et al. The AASM Manual for the Scoring of Sleep and Associated Events: Rules, Terminology, and Technical Specification (1. Aufl.). Westchester, IL.: American Academy of Sleep Medicine 2007

[17] Ingersoll C. Editorial sleep efficiency and overreaching in swimmers. Journal of Sports Rehabilitation 2003; 12: 1-12

[18] Johns MW. A new method for measuring daytime sleepiness: The Epworth Sleepiness Scale. Sleep 1991; 14: 540-545

[19] Juliff LE, Halson SL, Pfeiffer HJJ. Understanding sleep disturbance in athletes prior to important competitions. Journal of Science and Medicine in Sport 2014; 18: 13-18

[20] Kallus KW. Erholungs-Belastungs-Fragebogen: EBF. Frankfurt, Germany: Swets Test Services 1995 
[21] King BR, Hoedlmoser K, Hirschauer F et al. Sleeping on the motor engram: The multifaceted nature of sleep-related motor memory consolidation. Neuroscience \& Biobehavioral Reviews 2017; 80: 1-22

[22] Leatherwood WE, Dragoo JL. Effect of airline travel on performance: a review of the literature. $\mathrm{Br} J$ Sports Med 2012; bjsports-2012: 1-9

[23] Leeder J. Glaister M. Pizzoferro K et al. Sleep duration and quality in elite athletes measured using wristwatch actigraphy. Journal of Sports Sciences 2012; 30: 541-545

[24] Maddox WT, Glass BD, Wolosin SM et al. The effects of sleep deprivation on information-integration categorization performance. Sleep 2009; 32: 1439-1448

[25] Mah C. Sleep to be an all-star. (Infographic). Zoe, Inc 2011

[26] Mah C, Mah K, Dement W. Extended sleep and the effects on mood and athletic performance in collegiate swimmers. Sleep 2008; 31: A128, Abstract 0384

[27] Mah C, Mah K, Dement W. Athletic performance improvements and sleep extension in collegiate tennis players. Sleep 2009; 32: A155, Abstract 0469

[28] Mah C, Mah K, Dement W. Sleep extension and athletic performance in collegiate football. Sleep 2010; 33: A105-A106, Abstract 0304

[29] Martin B]. Effect of sleep deprivation on tolerance of prolonged exercise. European Journal of Applied Physiology and Occupational Physiology 1981; 47: 345-354

[30] Martin B], Gaddis GM. Exercise after sleep deprivation. Medicine and Science in Sports and Exercise 1980; 13: 220-223

[31] Matos NF, Winsley RJ, Williams CA. Prevalence of nonfunctional overreaching/overtraining in young English athletes. Medicine and Science in Sports and Exercise 2011; 43: 1287-1294

[32] Oda S, Shirakawa K. Sleep onset is disrupted following presleep exercise that causes large physiological excitement at bedtime. European Journal of Applied Physiology 2014; 114: 1789-1799

[33] Rechtschaffen A. The control of sleep. In W. A. Hunt (Ed.), Human behavior and its control 1971: 75-92 MA: Schenkman

[34] Reilly T, Piercy M. The effect of partial sleep deprivation on weight-lifting performance. Ergonomics 1994; 37: 107-115

[35] Roth K, Erlacher D, Binnig D et al. Wechselwirkungen zwischen Schlaf und Leistung im Hochleistungsradsport. 2009 Entnommen 2017: http://www.bisp.de/SharedDocs/ Downloads/Publikationen/Jahrbuch/Jb_200910_Artikel/ Roth_219_222.pdf?_blob=publicationFile.
[36] Sargent C, Halson S, Roach GD. Sleep or swim? Early-morning training severely restricts the amount of sleep obtained by elite swimmers. European Journal of Sport Science 2014; 14(sup1), S310-S315

[37] Sargent C, Schmidt WF, Aughey RJ et al. The impact of altitude on the sleep of young elite soccer players (ISA3600). Br J Sports Med 2013; 47(Suppl 1): i86-i92

[38] Savis JC. Sleep and athletic performance: Overview and implications for sport psychology. The Sport Psychologist 1994; 8: 111-125

[39] Silva A, Queiroz SS, Winckler C et al. Sleep quality evaluation, chronotype, sleepiness and anxiety of Paralympic Brazilian athletes: Beijing 2008 Paralympic Games. British Journal of Sports Medicine 2010; bjsports 77016

[40] Sleep in America poll: Exercise and sleep. Arlington (VA): National Sleep Foundation; 2013. http://sleepfoundation. org/sites/default/files/RPT336\%20Summary\%20of\%20Findings\%2002\%2020\%202013.pdf

[41] Spiegel K, Leproult R, Van Cauter E. Impact of sleep debt on metabolic and endocrine function. The Lancet 1999; 354: 1435-1439

[42] Steininger K, Wodick RE. Einfluß eines Schlaftherapeutikums (Brotizolam) auf die körperliche Leistungsfähigkeit und das Reaktionsvermögen von Leistungssportlern. Deutsche Zeitschrift für Sportmedizin 1987; 38: 208-212

[43] Stuck BA, Maurer JT, Schredl M et al. Praxis der Schlafmedizin 2009. Springer

[44] Taylor SR, Rogers GG, Driver HS. Effects of training volume on sleep, psychological, and selected physiological profiles of elite female swimmers. Medicine and Science in Sports and Exercise 1997; 29: 688-693

[45] Tietzel A], Lack LC. The recuperative value of brief and ultra-brief naps on alertness and cognitive performance. Journal of Sleep Research 2002; 11: 213-218

[46] Venter RE. Role of sleep in performance and recovery of athletes: A review article. South African Journal for Research in Sport, Physical Education and Recreation 2012; 34: 167-184

Bibliografie

DOI https://doi.org/10.1055/s-0043-123864 sportphysio 2018; 6: 16-23

(c) Georg Thieme Verlag KG Stuttgart · New York ISSN 2196-5951 